\title{
Numerical Research of Additional Mass Based on Panel Method
}

\author{
ZHANG Hao-jian ${ }^{1, a}$, ZHANG Jia-ning ${ }^{2, b}$ ZHANG Lei ${ }^{3, c}$ Li Tao $^{4, d}$ \\ ${ }^{1,2,3,4}$ Transportation Equipment and Ocean Engineering College, \\ Dalian Maritime University, Dalian 116026, China \\ a zhanghj10@163.com b zhangjianing@dlmu.edu.cn \\ ccsylzl@163.com ditaopostgrduate@sina.com
}

Key words: panel method; grid generation; additional mass; ellipsoid

\begin{abstract}
This paper is based on the panel method from three-dimensional potential flow theory, sources-sinks are distributed on surface panel to meet the boundary conditions. Additional mass can be obtained by solving the velocity potential. Ellipsoids under different scales are as example to study influence of grid generation on additional mass through various longitudinal and vertical grid numbers. By comparing the calculated values with the theoretical values, the way of grid generation and the number of panel that most satisfy the accuracy requirements can be obtained.
\end{abstract}

\section{Introduction}

Potential flow theory in fluid mechanics is a direct way to solve forces from fluid to object. By solving the Laplace equation the velocity potential of flow field could be obtained, and fluid force on object could be obtained through the Bernoulli equation[1,2]. As a very effective method of hydrodynamic analysis, the source-sink distribution method[3,4], also called constant panel method, is used to solve this problem. In order to obtain the velocity potential, the object plane and the free surface (or other boundary) must be divided to discrete grids[5,6,7,8]. Typically the object plane is divided to triangular or quadrilateral grid and the free surface to quadrilateral grid. with the increase in the number of the original surface, the geometric similarity and hydrodynamic similarity will be greatly improved through high-quality grid and sufficient grid numbers. Although the increase in the number of surface panel is good for accuracy, there will not be a great improvement in the accuracy while the number of surface panel is to a certain extent. Furthermore, the number of surface panel should be within a certain range as its disadvantages of long time-consuming in numerical calculation. In this paper the ellipsoids under different scales ratio are chosen as the object, influence of their grid generation on additional mass through various longitudinal and vertical grid numbers will be studied.

\section{Principle and Mathematical Model}

\section{The establishment of the coordinate system}

Coordinate system meets the Right-hand Rule, as shown in Fig.1, coordinate system origin $o$ is located in the center of the ellipsoid volume, axis $\mathrm{X}$ points to the head-end along major axis of ellipsoid, axis Y points to the left of the ellipsoid, axis $\mathrm{Z}$ is perpendicular to the plane xoy and points upward.

\section{Equations and boundary conditions}

Assuming that flow is incompressible, inviscid and irrotational and the object is in the plane 
with a steady linear motion, the perturbation velocity potential represents as $\Phi=\sum_{i=1}^{6} u_{i} \varphi_{i}$, Where $\mathrm{u}_{1} \sim \mathrm{u}_{6}$ correspond respectively to the speed under six degrees of freedom; $\varphi_{i}$ corresponds to perturbation velocity potential under object modal $i$ motion with unit speed. The definite conditions is as follows:

$$
\begin{array}{ll}
\nabla \varphi_{i}=0 & \text { (inside the flow field) } \\
\frac{\partial \varphi_{i}}{\partial \mathrm{n}}=n & \text { (on the object surface) } \\
\varphi_{i} \rightarrow 0 & \text { (Infinity) }
\end{array}
$$

Where, $\boldsymbol{n}$ is unit normal vector on the object surface (pointing to inboard ).

Then the additional mass can be calculated by formula, as follows:

$$
m_{j i}=\rho \iint_{S_{b}} \varphi_{j} \frac{\partial \varphi_{i}}{\partial n} d S \quad(i, j=1,2, \mathrm{~L}, 6)
$$

Where $S_{b}$ is surface area of object; $\rho$ is fluid density.

The influence of free surface wave is not taken account into the establishment of the velocity potential conditions and the calculation of the additional mass, so the additional mass is not related to the speed. And the study that the influence of different object surface grid on the additional mass is major task in this paper, the impact of the free surface is not considered temporarily.

\section{Equation solution method}

Supposing that $q$ is the source point on object surface, $p$ is one point in the flow field, $r(p, q)$ represents the distance between $q$ and $p, \sigma(q)$ represents the source density of distribution on the surface, then perturbation velocity potential of any point $p$ in the flow field, it is as follows:

$$
\varphi(p)=\iint_{S} \frac{\sigma(q)}{r(p, q)} d S
$$

Eq.3 is combined with the second equation of Eq.1, it is as follows:

$$
2 \pi \sigma(q)+\iint_{S-\varepsilon} \sigma(q) \frac{\partial}{\partial n_{p}}\left(\frac{1}{r(p, q)}\right) d S_{q}=n_{p}
$$

Where $S_{q}$ represents body-surface panel at point $q$, $\varepsilon$ represents a surface panel near control point $p$.

The integral term is usually converted into a linear algebraic sum. Object surface $S_{b}$ can be divided into $\mathrm{N}$ discrete pieces, $S_{b}=\sum_{j=1}^{N} \Delta S_{j}$.

Then small curved surface $\triangle S_{j}$ will be replaced approximately with triangular or quadrangular plane $\triangle \mathrm{Q}_{\mathrm{j}}$, Specifically as follows: in case of the piece $j$, arithmetic mean of four vertex coordinates is coordinate of the center point $p_{j}$. The cross product of two diagonal vectors can be calculated, this cross product can be regarded as the normal vector $\boldsymbol{n}_{\mathrm{j}}$ in piece $j$. And a vertical plane can be obtained from the normal vector $\boldsymbol{n}_{\mathrm{j}}$ and the center point $p_{j}$, four projected points can be obtained after the four vertices are projected to this plane. quadrangular plane $\triangle \mathbf{Q}_{j}$, combined with 
four projected points, is needed[9]. If division of the surface panel is not be too rough,distributed source density $\sigma(q)$ on surface panel $\triangle \mathrm{Q}_{\mathrm{j}}$ can be regarded as a constant. So integral on the full surface $S_{b}$ can be replaced by Sum of $\mathrm{N}$ integrals on quadrangular plane (or triangle plane) $\triangle \mathrm{Q}_{\mathrm{j}}$, it is as follows:

Eq.5

$$
\iint_{S} \sigma(q) \frac{\partial}{\partial n_{p}}\left(\frac{1}{r(p, q)}\right) d S_{q} \approx \sum_{j=1}^{N} \sigma_{i} \iint_{\Delta Q_{j}} \frac{\partial}{\partial n_{p}}\left(\frac{1}{r(p, q)}\right) d Q_{j}
$$

is combined with Eq.4, $\sigma_{\mathrm{j}} \mathrm{N}$-order linear equations can be obtained:

$$
\sum_{j=1}^{N} a_{i j} \sigma_{j}=b_{i} \quad(i=1,2, \mathbf{L}, N)
$$

Where $\mathrm{a}_{\mathrm{ij}}$ is regarded as influence coefficient,

$$
\begin{aligned}
& a_{i j}= \begin{cases}\iint_{\Delta Q_{j}} \frac{\partial}{\partial n_{p}}\left(\frac{1}{r(p, q)}\right) d Q_{j} & (i \neq j) \\
2 \pi & (i=j)\end{cases} \\
& b_{i}=n_{p i}
\end{aligned}
$$

\section{Algorithm analysis}

The ellipsoid is considered as an example, $a$ is the long half axis, $b$ is short half axis and $c$ is the vertical short half axis. The influence of the grid division on the added mass was studied at four scale ratios of $a: b: c=1: 1: 1, a: b: c=2: 1: 1, a: b: c=5: 1: 1$, and $a: b: c=10: 1: 1$. K1 indicates the number of longitudinal gird, and K2 is the vertical grid number. This research on the added mass of surge $m_{11}$ was undertaken, finally compared the calculated value with the theoretical value [10] and analyzed the results.

The ellipsoid becomes a sphere when the scale ratio is $a: b: c=1: 1: 1$ (Fig.1). Fig.2 shows the calculation error of $m_{11}$ with the variable number of the longitudinal and vertical grid. The vertical mesh number has a great impact on $m_{11}$ and the longitudinal grid number denotes the opposite effect.

When the scale ratio is $a: b: c=2: 1: 1$, from Fig.3, it show the same variable trend of $m_{11}$ as Fig.2. The vertical grid number has a great impact on $m_{11}$ and the longitudinal grid number denotes the opposite effect when length-width ratio of grid is not large. 


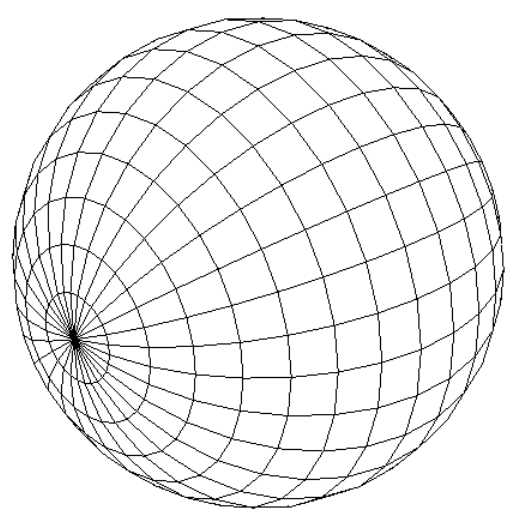

Fig.1 Sphere $(a: b: c=1: 1: 1)$

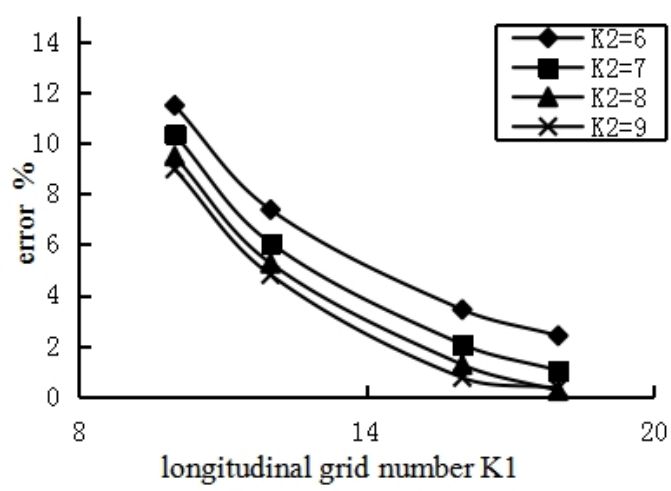

Fig.3 Calculation errors $(a: b: c=2: 1: 1)$

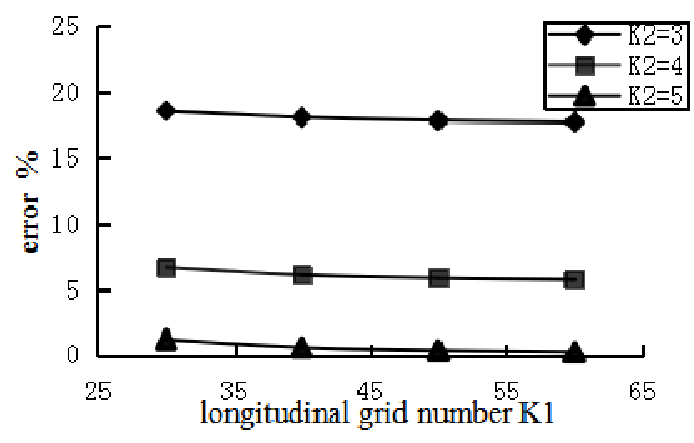

Fig.5 Calculation errors $(a: b: c=10: 1: 1)$

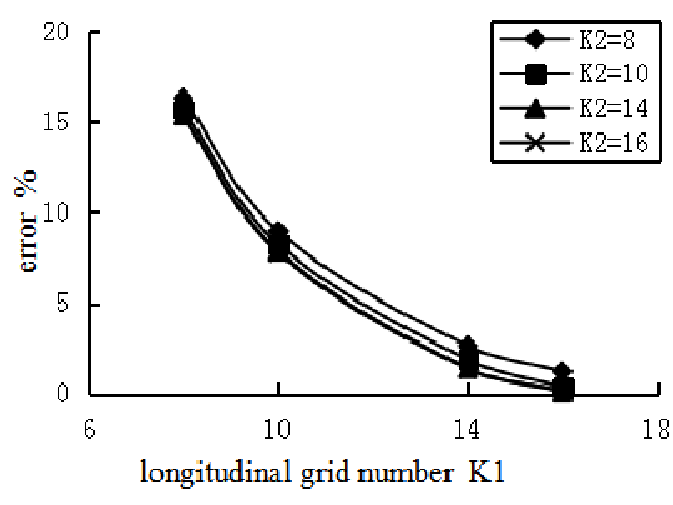

Fig.2 Calculation errors $(a: b: c=1: 1: 1)$

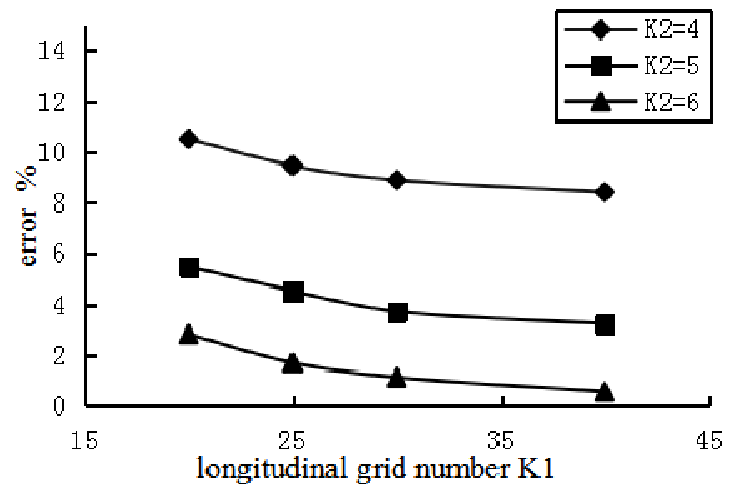

Fig.4 Calculation errors $(a: b: c=5: 1: 1)$

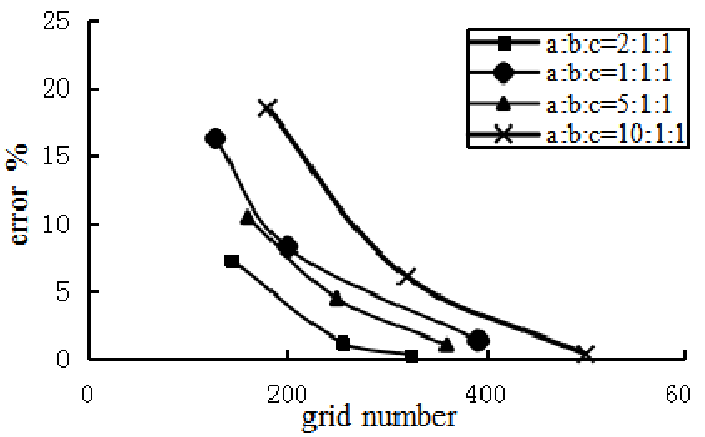

Fig.6 Calculation errors (K1:K2=1)

When the scale ratio of $a: b: c=5: 1: 1$, from Fig.4, the beneficial increase in the number of longitudinal grid slightly reduce calculation errors of $m_{11}$; with the increase in the number of the vertical grid, the calculation accuracy of $m_{11}$ shows a large degree of improvement. With the increase in the total number of panels, the error between $m_{11}$ calculated and theoretical values is getting smaller which is in line with the actual situation.

The ellipsoid becomes a slender body when the scale ratio of $a: b: c=10: 1: 1$, from Fig.5, it and Fig. 4 is roughly the same as the number of the longitudinal grid increase the accuracy of $m_{11}$ with little improvement, but the increasing of vertical grid number is benefit to reduce the error of $m_{11}$. The error between $m_{11}$ calculated and theoretical values is reduced with the increase in the total panel number. 


\section{Conclusions}

The conclusions obtained from the above analysis are as follows:

(1) The calculation accuracy of additional mass increases with the number of panels(Fig.6).

(2) When the aspect ratio of object is not too large, $m_{11}$ is greatly influenced by the longitudinal division and slightly influenced by the vertical division; When the aspect ratio becomes larger, the vertical division greatly influences $m_{11}$ and the longitudinal division influences $m_{11}$ on the contrary.

(3) Grid generation should be adapt to the scale of the object, an appropriate longitudinal and vertical mesh division can improve the calculation accuracy and reduce the computational time.

\section{Acknowledgements}

This work was financially supported by National and International Scientific and Technological Cooperation Special Projects(2013DFA80760) and the Fundamental Research Funds for the Central Universitie(01760619)

\section{References}

[1] L. Zhang, Y.B. Li. Fluid Mechanics. Harbin Engineering University Press (2009)

[2] R.H. Zhang. Wave Load Calculation Based Linear Potential Flow Theory and Its Application. South China University of Technology (2011)

[3] X.Y. Sun, J.Z. Song and Y. Lin. Source-sink Distribution Method for Solving Hydrodynamic Coefficients of Asymmetrical Section. Dalian Maritime University Press, Vol. 02(2006), p.106-110

[4] D.X. Zhu, J.P. Chen. Application of Panel Method Based on Rankine Source in the Optimization of Hull Form. Proceedings of the Ninth National Conference on Hydrodynamics and the Twenty-second National Symposium on Hydrodynamics(2009), p.779-788

[5] X.J. Lv, X.G. Wu and J.L. Sun. Trim Optimization of Ship by a Potential-Based Panel Method. Advances in Mechanical Engineering(2013)

[6] Z.R. Li. The Prediction of Ship Wave-making Resistance by Panel Method. Wuhan University of Technology(2006)

[7] Y.Z. Dai, J.X. Yu. 3D Mesh Auto-generating Method of Ship Hull and Free Surface. Ship Engineering. Vol. 28 (2006), p.1-4

[8] R.B. Inglis. A Three Dimensional Analysis of the Motion of a Rigid Ship in Waves. Unversity of London(1980)

[9] Y.S. Dai. Frequency-domain and Time-domain Potential Flow Theory of Ship Motion in Waves. National Defence Industry Press, Beijing(1998)

[10] C. Farell. On the Wave Resistance of a Submerged Spheroid. JSR, Vo1.17 (1973), p.1 11 